\title{
ZETA-FUNCTION OF A NONLINEAR SYSTEM
}

\author{
A. KYTMANOV, S. MYSLIVETS, AND N. TARKHANOV
}

\begin{abstract}
Given a system of entire functions in $\mathbb{C}^{n}$ with at most countable set of common zeros, we introduce the concept of zeta-function associated with the system. Under reasonable assumptions on the system, the zeta-function is well defined for all $s \in \mathbb{Z}^{n}$ with sufficiently large components. Using residue theory we get an integral representation for the zeta-function which allows us to construct an analytic extension of the zeta-function to an infinite cone in $\mathbb{C}^{n}$.
\end{abstract}

\section{Contents}

1. Introduction

2. Zeta-function of elliptic operators 2

3. Generalised zeta-function 4

4. Preliminary results 4

5. An integral representation $\quad 6$

6. Proper systems 8

7. An example 9

References

\section{INTRODUCTION}

In the present paper we define a zeta-function associated with a system of nonlinear equations in $\mathbb{C}^{n}$. As but one motivation of this concept we mention numerous applications in the elimination theory for nonlinear systems and in algebraic geometry, cf. [AYu83], [BKL98], [Tsi92], etc.

The basis for the solution of systems of algebraic equations is the method of elimination of unknowns. As a rule, the procedure for elimination is realised by the successive elimination of each of the unknowns, using Sylvester's resultant of two polynomials.

If the original system has only isolated roots, then at the last stage of the elimination we obtain a system of equations in one unknown, which we can replace by one equation. In case the number of equations just amounts to the number of unknowns, the multidimensional logarithmic residue allows one to eliminate all of the unknowns except one at one step. The resulting equation preserves the multiplicity of the roots.

Date: October 25, 2004.

2000 Mathematics Subject Classification. Primary 11Mxx; Secondary 32A60.

Key words and phrases. Nonlinear systems, zeta-function, residue. 
The construction of the resulting equation is suitable also for the solution of systems of holomorphic equations.

Suppose that $f=\left(f_{1}, \ldots, f_{n}\right)$ is a system of holomorphic functions in a domain $\mathcal{D} \subset \mathbb{C}^{n}$, which has finitely many zeros $\left\{a_{k}\right\}$ in $\mathcal{D}$. After [Tsi92, $\left.\S 19\right]$, the resultant of a function $F: \mathcal{D} \rightarrow \mathbb{C}$ with respect to the system $f$ is the number

$$
R_{f}(F)=\prod_{k}\left(F\left(a_{k}\right)\right)^{\mu_{k}},
$$

where $\mu_{k}$ is the multiplicity of the zero $a_{k}$ of the system $f$. In the case when $n=1, \mathcal{D}=\mathbb{C}$, and $f$ and $F$ are polynomials, the resultant $R_{f}(F)$ coincides with Sylvester's resultant. Taking the logarithm of both sides of the equality (1.1) easily gives

$$
\log R_{f}(F)=\sum_{a \in N_{f}} \log F(a)
$$

where the sum is over all zeros of $f$ in $\mathcal{D}$ counted with their multiplicities. The right-hand side is well defined if the image of $N_{f}$ by $F$ lies in $\mathbb{C}$ with slit along a ray from the origin.

When considering a system of holomorphic functions $f$ in a product domain $\mathcal{D}=\mathcal{D}^{\prime} \times B$ of $\mathbb{C}^{n+1} \times \mathbb{C}$, one can introduce the resultant of the function $f_{n}\left(z^{\prime}, z_{n}\right)$ with respect to the truncated system $f^{\prime}\left(z^{\prime}, z_{n}\right)=\left(f_{1}\left(z^{\prime}, z_{n}\right), \ldots, f_{n-1}\left(z^{\prime}, z_{n}\right)\right)$, for fixed $z_{n} \in B$. The function $R\left(z_{n}\right)=R_{f^{\prime}}\left(f_{n}\right)$ obtained in this manner has the property that $R\left(z_{n}\right)=0$ if and only if the system $f\left(z^{\prime}, z_{n}\right)=0$ has a solution for this $z_{n}$.

If $f$ is a polynomial map of $\mathbb{C}^{n}$, then the resultants of the functions $F(z)=\exp z^{s}$, $s \in \mathbb{Z}_{+}^{n}$, with respect to $f$ are of crucial importance for the elimination theory. Indeed, they alow one, when combined with the Newton recurrence formulas, to explicitly evaluate $R\left(z_{n}\right)$, cf. $\S 8$ of [BKL98]. The series $\sum_{a \in N_{f}} a^{s}$ is an analogue of the Riemann zeta-function.

We introduce the zeta-function $\zeta_{f}(s)$ of the system $f$ by the series $\sum_{a \in N_{f}} a^{-s}$, if it converges. Note that $\zeta_{f}(s)$ is, by the very definition, a function of $n$ variables $s=\left(s_{1}, \ldots, s_{n}\right)$.

We are interested in the analyticity of $\zeta_{f}$ in $s \in \mathbb{C}^{n}$, which certainly depends on the location of the zero set of $f$. To highlight the situation, we recall the construction of zeta-function for an elliptic boundary problem.

\section{ZETA-FUnCTION OF ELLIPTIC OPERATORS}

The idee of this paper goes at least as far as [GL53]. This latter deals with the regularised trace for the Sturm-Liouville operator. Let $\left\{\lambda_{k}\right\}$ be the characteristic values of

$$
\begin{aligned}
-u^{\prime \prime}+q(x) u & =\lambda u \text { for } x \in(0, \pi), \\
u^{\prime}(0)-h u(0) & =0 \\
u^{\prime}(\pi)+H u(\pi) & =0
\end{aligned}
$$

the mean value of $q$ on the interval $[0, \pi]$ being zero. Denote by $\left\{c_{k}\right\}$ the characteristic values of $-u^{\prime \prime}=c u$ with the same conditions. Gelfand and Levitan proved in [GL53] that

$$
\sum_{k=1}^{\infty}\left(\lambda_{k}-c_{k}\right)=\frac{1}{4}(q(0)+q(\pi))+h H .
$$


The paper [Dik55] shed some new light on this formula. Let $\left\{\lambda_{k}\right\}$ be the eigenvalues of the same differential operator but with the Dirichlet boundary conditions $u(0)=u(\pi)=0$. From the identity

$$
\sum_{k=1}^{\infty}\left(\lambda_{k}+z\right)^{-1}=\operatorname{Tr}\left(D^{2}+q(x)+z\right)^{-1}
$$

one can obtain the formulas

$$
\sum_{k=1}^{\infty} \lambda_{k}^{n}=\operatorname{Tr}\left(D^{2}+q(x)+z\right)^{n},
$$

for $n=1,2, \ldots$, by expanding formally in powers of $z^{-1}$ and equating coefficients. The apparently meaningless formulas (2.2) can be "regularised" by forming asymptotic expansions of both sides of (2.1) for large $z$, assuming $q(x)$ to be infinitely differentiable.

In [Lev64] a new method is presented for calculation of the regularised sums $\sum_{k=1}^{\infty} \lambda_{k}^{s}$ with $s=1,2, \ldots$. It is based on specifying $\lambda_{k}$ as roots of an entire analytic function of $\lambda \in \mathbb{C}$.

An approach to perturbation theory due to Dikii [Dik55] is based on a study of Dirichlet series related to the positive eigenvalues of a selfadjoint operator. A problem of the theory is to sum divergent series of the form $\sum_{k} z_{k}^{n}$, where $n$ is a positive integer and the numbers $\left\{z_{k}\right\}$ are zeros of an entire function $f$. The sum is defined by analytic continuation of the corresponding Dirichlet series $\sum_{k} z_{k}^{-s}$. A solution is required from a knowledge of coefficients in an asymptotic expansion of $f$.

Lidskii and Sadovnichii studied in [LS67] a class of entire analytic functions $f$ of the form

$$
f(z)=\sum_{j=1}^{N} e^{c_{j} z} p_{j}(z)
$$

where $c_{j}$ are complex constants and each $p_{j}(z)$ has an asymptotic expansion of the form $p_{j}(z) \sim \sum_{k=-k_{j}}^{\infty} C_{j k} z^{-k}$ with $C_{j,-k_{j}} \neq 0$. It is assumed that the $z$-plane can be divided into a finite number of sectors, in each sector the $p_{j}(z)$ are analytic for large $|z|$ and the corresponding asymptotic expansions can be differentiated. Denote by $\left\{z_{k}\right\}$ the zeros of a function $f$ in the class and define the corresponding zeta-function $\zeta_{f}(s)=\sum_{k} z_{k}^{-s}$ in the half-plane $\Re s>1$. In [LS67], asymptotic expansions for $z_{k}$ are obtained and, by putting in certain convergence factors, the sum $\zeta_{f}$ is converted into a sum which converges for $\Re s>-m-1, m$ being any positive integer.

For a general elliptic boundary problem $A u=f$ with homogeneous boundary conditions on a compact $C^{\infty}$ manifold $M$ with boundary, the $\zeta$-function is defined by

$$
\zeta_{A}(s)=\int_{M} \operatorname{tr} K_{A^{s}}(x, x),
$$

where $K_{A^{z}}$ is the Schwartz kernel of the power $A^{z}$. Seeley proved in [See69] that the $\zeta$-function of an elliptic boundary problem has a meromorphic extension to all of $\mathbb{C}$.

If the boundary value problem is selfadjoint and $\left\{\lambda_{k}\right\}$ are its eigenvalues, then $\zeta_{A}(s)=\sum_{k} \lambda_{k}^{s}$ for $\Re s<-n / m$, where $n$ is the dimension of $M$ and $m$ the order of 
$A$. Moreover, the series converges absolutely and uniformly in $s$ in any half-plane $\Re s<-n / m-\varepsilon$ with $\varepsilon>0$, cf. for instance [Shu87, § 13].

\section{Generalised zeta-FunCtion}

Let $f=\left(f_{1}, \ldots, f_{n}\right)$ a system of entire functions in $\mathbb{C}^{n}$. Consider the system of equations

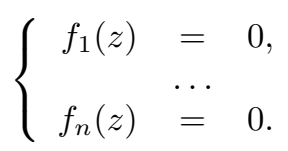

Denote by $N_{f}=f^{-1}(0)$ the set of all roots of system (3.1), every root being considered along with its multiplicity. From now on we assume that $N_{f}$ is at most countable. Hence it follows that this set has no accumulation points but the point at infinity. Indeed, take any bounded domain $D \subset \mathbb{C}^{n}$ whose boundary does not contain any roots of (3.1). Then $N_{f} \cap D$ is a compact analytic set in $D$, hence it is finite. It follows that $N_{f}$ is discrete, as desired.

We will be concerned with an integral representation for the zeta-function $\zeta_{f}(s)$ of system (3.1), i.e.,

$$
\zeta_{f}(s)=\sum_{a \in N_{f}} a^{-s}
$$

where $s=\left(s_{1}, \ldots, s_{n}\right)$ and $a^{-s}=a_{1}^{-s_{1}} \cdot \ldots \cdot a_{n}^{-s_{n}}$.

Note that the classical zeta-function of Riemann corresponds this way to the equation $\exp z=1$.

\section{Preliminary Results}

Write $z_{j}=x_{j}+\imath y_{j}$ for $j=1, \ldots, n$. Suppose no function $f_{j}$ vanishes on the set $\overline{\mathbb{R}}_{+}^{n}:=\left\{z \in \mathbb{C}^{n}: x_{1}, \ldots, x_{n} \geq 0, y_{1}=\ldots=y_{n}=0\right\}$. In particular, this implies $N_{f} \cap \overline{\mathbb{R}}_{+}^{n}=\emptyset$.

Let us choose a polyhedral domain $D$ of the form $D=D_{1} \times \ldots \times D_{n}$ in $\mathbb{C}^{n}$, where

$$
D_{j}=\left\{z \in \mathbb{C}: r_{j}<|z|<R_{j}\right\} \backslash\left\{z \in \mathbb{C}: r_{j}<\Re z<R_{j}, \Im z=0\right\}
$$

for $0<r_{j}<R_{j}$, cf. Fig. 1 .

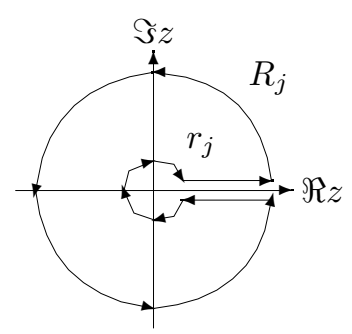

FIG. 1. The domain $D_{j}$.

Note that every $D_{j}$ is a simply connected domain. Its boundary $\Gamma_{j}=b D_{j}$ consists of the segment $\left[r_{j}, R_{j}\right]$ on the real axis, the circle $S_{R_{j}}$ of radius $R_{j}$ around the origin with positive orientation, the segment $\left[R_{j}, r_{j}\right]$ on $\mathbb{R}$ which just amounts 
to $\left[r_{j}, R_{j}\right]$ with opposite orientation, and the circle $-S_{r_{j}}$ which is equal to $S_{r_{j}}$ with opposite orientation.

The product $\Gamma=\Gamma_{1} \times \ldots \times \Gamma_{n}$ is the skeleton of $D$. We choose the radii $r_{j}$ and $R_{j}, j=1, \ldots, n$, in such a way that $\Gamma \cap N_{f}=\emptyset$.

Consider the integral

$$
\mathcal{I}(-s)=\frac{1}{(2 \pi \imath)^{n}} \int_{\Gamma}(-z)^{-s} \frac{d f}{f}
$$

where

$$
\frac{d f}{f}=\frac{d f_{1} \wedge \ldots \wedge d f_{n}}{f_{1} \cdot \ldots \cdot f_{n}}
$$

and $(-z)^{-s}=\left(-z_{1}\right)^{-s_{1}} \cdot \ldots \cdot\left(-z_{1}\right)^{-s_{n}}$. The functions $\left(-z_{j}\right)^{-s_{j}}=e^{-s_{j} \ln \left(-z_{j}\right)}$ are actually holomorphic in the domains $D_{j}$ if by $\ln \zeta$ is meant the main branch of the logarithm, i.e., the regular branch of $\ln \zeta$ in $\mathbb{C} \backslash\{\zeta \in \mathbb{C}: \Re \zeta \leq 0, \Im \zeta=0\}$ that vanishes at $\zeta=1$. It is easy to see that the integral $\mathcal{I}(-s)$ is an entire function of $s \in \mathbb{C}^{n}$.

Denote by $\left[f_{j}\right]=\left\{z \in \mathbb{C}^{n}: f_{j}(z)=0\right\}$ the divisor of the function $f_{j}$. We say that the domain $D$ agrees with the map $f$ if $\left[f_{j}\right] \cap(b D)_{j}=\emptyset$ for each $j=1, \ldots, n$, where

$$
(b D)_{j}=\bar{D}_{1} \times \ldots \times \bar{D}_{j-1} \times \Gamma_{j} \times \bar{D}_{j+1} \times \ldots \times \bar{D}_{n}
$$

is the $j$-th face of the boundary of $D$.

If $D$ agrees with $f$ then applying the principle of separating cycles [Tsi92, § 9.2] immediately yields

$$
\mathcal{I}(-s)=\sum_{a \in N_{f} \cap D}(-a)^{-s} .
$$

A trivial verification shows that the condition $\left[f_{j}\right] \cap(b D)_{j}=\emptyset$ can be rewritten in the form

1) $f_{j}(z) \neq 0$ if $\left|z_{j}\right|=r_{j}$ and $r_{k} \leq\left|z_{k}\right| \leq R_{k}$ for $k \neq j$;

2) $f_{j}(z) \neq 0$ if $\left|z_{j}\right|=R_{j}$ and $r_{k} \leq\left|z_{k}\right| \leq R_{k}$ for $k \neq j$;

3) $f_{j}(z) \neq 0$ if $r_{j} \leq \Re z_{j} \leq R_{j}$, ऽ $z_{j}=0$ and $r_{k} \leq\left|z_{k}\right| \leq R_{k}$ for $k \neq j$.

The formula (4.2) still remains valid when we drop the assumption 3). Indeed, consider an auxiliary system

$$
F_{j}(z)=0
$$

for $j=1, \ldots, n$, where $F_{j}(z)=f_{j}\left(0, \ldots, 0, z_{j}, 0, \ldots, 0\right)$. It satisfies the condition 3$)$ because $f_{j}\left(0, \ldots, 0, x_{j}, 0, \ldots, 0\right) \neq 0$ for all $x_{j} \geq 0$. The function $F_{j}(z)$ is obtained from $f_{j}(z)$ by equating the Taylor coefficients of the monomials containing a variable $z_{k}$ with $k \neq j$, to zero. Under sufficiently small perturbations of these coefficients the condition 3) remains still fulfilled, for the sets over which $z_{k}$ vary are compact. It follows that formula (4.2) holds for such coefficients. Since both the left-hand side and the right-hand side of (4.2) depend holomorphically on the Taylor coefficients of $f$ away from the discriminant set, we deduce that the formula (4.2) remains still true when we drop 3). 


\section{An integral REPRESENTATion}

Let us investigate the behaviour of $\mathcal{I}(-s)$ when $r_{j} \rightarrow 0$ and $R_{j} \rightarrow+\infty$, for $j=1, \ldots, n$. The skeleton $\Gamma$ splits into sets defined by means of circles $S_{r_{j}}, S_{R_{j}}$ and segments $\left[r_{j}, R_{j}\right]$ oriented in either of two directions.

Consider an integration set of the form $S_{r_{1}} \times \Gamma_{2} \times \ldots \times \Gamma_{n}$. Let us show that for $\Re s_{1}<1$ the integral

$$
\int_{S_{r_{1}}}\left(-z_{1}\right)^{-s_{1}} \frac{d f_{1}}{f_{1}} \wedge \int_{\Gamma_{2} \times \ldots \times \Gamma_{n}}\left(-z_{2}\right)^{-s_{2}} \cdot \ldots \cdot\left(-z_{n}\right)^{-s_{n}} \frac{d f_{2} \wedge \ldots \wedge d f_{n}}{f_{2} \cdot \ldots \cdot f_{n}}
$$

tends to zero if $r_{1} \rightarrow 0$. We get

$$
\begin{aligned}
\left|\left(-z_{1}\right)^{-s_{1}}\right| & =\left|e^{-s_{1}\left(\ln r_{1}+\imath\left(\varphi_{1}-\pi\right)\right)}\right| \\
& =e^{-\Re s_{1} \ln r_{1}+\Im s_{1}\left(\varphi_{1}-\pi\right)} \\
& =O\left(r_{1}^{-\Re s_{1}}\right)
\end{aligned}
$$

for $r_{1} \rightarrow 0$, where $\varphi_{1}=\arg z_{1}$. Since $f_{1}(0) \neq 0$, it follows that

$$
\begin{aligned}
\left|\int_{S_{r_{1}}}\left(-z_{1}\right)^{-s_{1}} \frac{d f_{1}}{f_{1}}\right| & \leq c \int_{S_{r_{1}}}\left|\left(-z_{1}\right)^{-s_{1}}\right|\left|d z_{1}\right| \\
& \leq C r_{1}^{1-\Re s_{1}}
\end{aligned}
$$

with constants $c$ and $C$ independent of $r_{1}$. Hence the first integral of (5.1) tends to 0 , when $r_{1} \rightarrow 0$, and so does the whole integral, as desired.

The same reasoning applies to any integral whose integration set includes at least one of $S_{r_{j}}$. All the integrals tend to zero, when $r_{j} \rightarrow 0$, provided $\Re s_{j}<1$.

We next consider the integral over the set $S_{R_{1}} \times \Gamma_{2} \times \ldots \times \Gamma_{n}$. We obviously have

$$
\int_{S_{R_{1}} \times \Gamma_{2} \times \ldots \times \Gamma_{n}}(-z)^{-s} \frac{d f}{f}=\int_{S_{R_{1}} \times \Gamma_{2} \times \ldots \times \Gamma_{n}}(-z)^{-s} \frac{J_{f}}{f} d z
$$

where $J_{f}$ is the Jacobian of the map $f$. If $\Re s_{1}>0$ and

$$
\frac{J_{f}}{f}=O\left(\frac{1}{R_{1}}\right)
$$

when $R_{1} \rightarrow+\infty$, then the integral (5.2) tends to 0 , when $R_{1} \rightarrow+\infty$, for

$$
\left|\int_{S_{R_{1}}}\left(-z_{1}\right)^{-s_{1}} \frac{J_{f}}{f} d z_{1}\right| \leq C R_{1}^{-\Re s_{1}}
$$

just as above.

If the condition

$$
\frac{J_{f}}{f}=O\left(\frac{1}{R_{j}}\right)
$$

when $R_{j} \rightarrow+\infty$, is fulfilled for all $j=1, \ldots, n$, then all the integrals whose integration set includes at least one of $S_{R_{j}}$ tend to zero.

It remains to consider the integrals over the product of segments oriented in diverse directions. We restrict our attention to a product

$$
P=\left[R_{1}, r_{1}\right] \times \ldots \times\left[R_{k}, r_{k}\right] \times\left[r_{k+1}, R_{k+1}\right] \times \ldots \times\left[r_{n}, R_{n}\right]
$$

where the first $k$ segments are oriented in the direction of decreasing the variable $x_{j}:=\Re z_{j}$. 
By the definition of the holomorphic function $(-z)^{-s}$, we immediately get the equality

$$
\int_{P}(-z)^{-s} \frac{d f}{f}=(-1)^{k} e^{-2 \pi \imath s_{1}} \cdot \ldots \cdot e^{-2 \pi \imath s_{k}} \int_{\left[r_{1}, R_{1}\right] \times \ldots \times\left[r_{n}, R_{n}\right]}(-x)^{-s} \frac{d f}{f}
$$

because

$$
\begin{aligned}
(-z)^{-s} & =\left(-x_{1} e^{2 \pi \imath}\right)^{-s_{1}} \cdot \ldots \cdot\left(-x_{k} e^{2 \pi \imath}\right)^{-s_{k}}\left(-x_{k+1}\right)^{-s_{k+1}} \cdot \ldots \cdot\left(-x_{n}\right)^{-s_{n}} \\
& =e^{-2 \pi \imath s_{1}} \cdot \ldots \cdot e^{-2 \pi \imath s_{k}}(-x)^{-s}
\end{aligned}
$$

Summing up the integrals over the sets which contain only segments we arrive at the integral

$$
\frac{1}{(2 \pi \imath)^{n}}\left(1-e^{2 \pi \imath\left(1-s_{1}\right)}\right) \cdot \ldots \cdot\left(1-e^{2 \pi \imath\left(1-s_{n}\right)}\right) \int_{\left[r_{1}, R_{1}\right] \times \ldots \times\left[r_{n}, R_{n}\right]}(-x)^{-s} \frac{d f}{f}
$$

as is easy to check.

Letting $r_{j} \rightarrow 0$ and $R_{j} \rightarrow \infty$ in (4.2) we thus get

$$
\frac{1}{(2 \pi \imath)^{n}}\left(1-e^{2 \pi \imath\left(1-s_{1}\right)}\right) \cdot \ldots \cdot\left(1-e^{2 \pi \imath\left(1-s_{n}\right)}\right) \int_{\overline{\mathbb{R}}_{+}^{n}}(-x)^{-s} \frac{d f}{f}=\sum_{a \in N_{f}}(-a)^{-s}
$$

the series on the right-hand side converges because so does the integral on the left-hand side. An easy computation gives

$$
\begin{aligned}
1-e^{2 \pi \imath\left(1-s_{j}\right)} & =e^{\pi \imath\left(1-s_{j}\right)} 2 \imath \frac{e^{-\pi \imath\left(1-s_{j}\right)}-e^{\pi \imath\left(1-s_{j}\right)}}{2 \imath} \\
& =-2 \imath e^{\pi \imath\left(1-s_{j}\right)} \sin \pi\left(1-s_{j}\right) \\
& =(-1)^{-s_{j}} 2 \imath \sin \pi\left(1-s_{j}\right),
\end{aligned}
$$

hence the left-hand side of (5.4) is equal to

$$
\frac{1}{\pi^{n}} \sin \pi\left(1-s_{1}\right) \cdot \ldots \cdot \sin \pi\left(1-s_{n}\right) \int_{\overline{\mathbb{R}}_{+}^{n}} x^{-s} \frac{d f}{f} .
$$

Summarising, we get an integral representation of the zeta-function $\zeta_{f}(s)$ in the product of critical strips $0<\Re s_{j}<1$.

Theorem 5.1. Suppose $0<\Re s_{j}<1$ for all $j=1, \ldots, n$, and (5.3) is satisfied. Then,

$$
\frac{1}{\pi^{n}} \sin \pi\left(1-s_{1}\right) \cdot \ldots \cdot \sin \pi\left(1-s_{n}\right) \int_{\overline{\mathbb{R}}_{+}^{n}} x^{-s} \frac{d f}{f}=\sum_{a \in N_{f}}(-a)^{-s} .
$$

Set $\mathcal{C}_{j}=\left(+\infty, r_{j}\right] \cup\left(-S_{r_{j}}\right) \cup\left[r_{j},+\infty\right)$ and $\mathcal{C}=\mathcal{C}_{1} \times \ldots \times \mathcal{C}_{n}$. The proof of Theorem 5.1 actually shows that

$$
\frac{1}{(2 \pi \imath)^{n}} \int_{\mathcal{C}} x^{-s} \frac{d f}{f}=\sum_{a \in N_{f}}(-a)^{-s} .
$$

The integral in (5.6) converges if $\Re s_{j}>0$ for each $j=1, \ldots, n$. It thus gives an analytic extension of the zeta-function $\zeta_{f}(a)$ to the product of half-planes $\Re s_{j}>0$. 


\section{Proper Systems}

The condition (5.3) is always fulfilled for polynomials $f_{j}$, and Theorem 5.1 holds in this case.

We now assume that each function $f_{j}$ can be written as infinite product of polynomials, i.e.,

$$
f_{j}(z)=\prod_{k=1}^{\infty} p_{j k}(z)
$$

for $j=1, \ldots, n$.

Corollary 6.1. Assume that $0<\Re s_{j}<1$ is fulfilled for $j=1, \ldots, n$, and the integral

$$
\int_{\overline{\mathbb{R}}_{+}^{n}} x^{-s} \frac{d f}{f}
$$

converges absolutely. Then formula (5.5) holds for system (6.1).

Proof. An elementary computation gives

$$
\begin{aligned}
\frac{d f}{f} & =\frac{d \prod_{k=1}^{\infty} p_{1 k}}{\prod_{k=1}^{\infty} p_{1 k}} \wedge \ldots \wedge \frac{d \prod_{k=1}^{\infty} p_{n k}}{\prod_{k=1}^{\infty} p_{n k}} \\
& =\sum_{k_{1}, \ldots, k_{n}=1}^{\infty} \frac{d p_{1 k_{1}}}{p_{1 k_{1}}} \wedge \ldots \wedge \frac{d p_{n k_{n}}}{p_{n k_{n}}} .
\end{aligned}
$$

Note that formula (5.5) is true for each system of polynomials $p_{1 k_{1}}, \ldots, p_{n k_{n}}$, which is due to Theorem 5.1. Integrating term by term the series obtained establishes the corollary.

We now discuss some other classes of functions which satisfy the conditions of Theorem 5.1. Let $f_{1}(z)=f_{1}\left(z_{1}\right)$ be a polynomial satisfying $f_{1}(0) \neq 0$. Then $f_{1}\left(z_{1}\right)$ does not vanish for $\left|z_{1}\right|$ small enough. Clearly, $f_{1}\left(z_{1}\right)$ is different from 0 for sufficiently large $\left|z_{1}\right|$. Moreover, $f_{1}\left(x_{1}\right) \neq 0$ for all $x_{1} \geq 0$, if for instance the coefficients of $f_{1}$ have positive real parts. Pick $r_{1}$ and $R_{1}$ satisfying the conditions 1) and 2) of Section 4. Take

$$
f_{2}(z)=c_{2,0}+\sum_{k=1}^{m_{2}} c_{2, k}\left(z_{1}\right) z_{2}^{k},
$$

where $c_{2,0}$ is a non-zero constant and $c_{2, k}\left(z_{1}\right)$ are entire functions of $z_{1}$ with $c_{2, m_{2}}\left(z_{1}\right) \neq 0$ in $\mathbb{C}$. Then $f_{2}(z)$ does not vanish if $r_{1} \leq\left|z_{1}\right| \leq R_{1}$ and $\left|z_{2}\right|$ is sufficiently small or sufficiently large. Hence there are $r_{2}$ and $R_{2}$ satisfying the conditions 1) and 2) of Section 4 . Once again we can choose $f_{2}$ which is different from zero on $\overline{\mathbb{R}}_{+}^{n}$, and so on. By induction,

$$
f_{n}(z)=c_{n, 0}+\sum_{k=1}^{m_{n}} c_{n, k}\left(z_{1}, \ldots, z_{n-1}\right) z_{n}^{k}
$$

where $c_{n, 0} \neq 0$ is a constant and $c_{2, k}\left(z_{1}, \ldots, z_{n-1}\right)$ are entire functions of $z_{1}$ with $c_{2, m_{n}}\left(z_{1}, \ldots, z_{n-1}\right) \neq 0$ in $\mathbb{C}^{n-1}$. Having fixed $r_{j}$ and $R_{j}$ for $j=1, \ldots, n-1$, one can choose $r_{n}$ and $R_{n}$, such that the conditions 1) and 2) of Section 4 are fulfilled. Moreover, one can guarantee that $f_{n}(z) \neq 0$ on all of $\overline{\mathbb{R}}_{+}^{n}$. It follows that the system $f=\left(f_{1}, \ldots, f_{n}\right)$ constructed in this manner satisfies the conditions 1$\left.)-3\right)$ of Section 
4. Our next goal is to show that the condition (5.3) is satisfied. To this end, we observe that

whence

$$
J_{f}=\left(f_{1}\right)_{z_{1}}^{\prime} \cdot \ldots \cdot\left(f_{n}\right)_{z_{n}}^{\prime}
$$

$$
\frac{J_{f}}{f}=\frac{\left(f_{1}\right)_{z_{1}}^{\prime}}{f_{1}} \cdot \ldots \cdot \frac{\left(f_{n}\right)_{z_{n}}^{\prime}}{f_{n}}
$$

Since $f_{j}$ is a polynomial in $z_{j}$ and the coefficient of the highest power of $z_{j}$ is bounded away from zero, we conclude that

$$
\frac{\left(f_{j}\right)_{z_{j}}^{\prime}}{f_{j}}=O\left(\frac{1}{R_{j}}\right)
$$

uniformly in the other variables $z_{1}, \ldots, z_{n-1}$, when $R_{j} \rightarrow+\infty$. Therefore, $f$ fulfills (5.3).

\section{An example}

Let $f_{1}, \ldots, f_{n}$ be polynomials with non-negative coefficients, such that the highest degree homogeneous parts of $f_{j}$ have a unique common zero at $0 \in \mathbb{C}^{n}$, and $f_{j}(0) \neq 0$ for $j=1, \ldots, n$. Then the system

$$
\left\{\begin{aligned}
f_{1}(z) & =c_{1} \\
f_{n}(z) & \cdots
\end{aligned}\right.
$$

has a finite number of roots in $\mathbb{C}^{n}$ for all constant numbers $c_{1}, \ldots, c_{n}$.

Consider the integral

$$
\begin{aligned}
I(s) & =\int_{\overline{\mathbb{R}}_{+}^{n}} x^{-s} \frac{d f_{1} \wedge \ldots \wedge d f_{n}}{\sinh f_{1} \cdot \ldots \cdot \sinh f_{n}} \\
& =\int_{\overline{\mathbb{R}}_{+}^{n}} x^{-s} \frac{d \tanh \frac{f_{1}}{2} \wedge \ldots \wedge d \tanh \frac{f_{n}}{2}}{\tanh \frac{f_{1}}{2} \cdot \ldots \cdot \tanh \frac{f_{n}}{2}} .
\end{aligned}
$$

Obviously, the integral $I(s)$ converges absolutely if $\Re s_{j}<1$ for all $j=1, \ldots, n$. Hence, Corollary 6.1 applies to $I(s)$, the only difference being in the meromorphy of $\tanh \left(f_{j} / 2\right)$. As

$$
\begin{aligned}
\sinh \frac{f_{j}}{2} & =\frac{f_{j}}{2} \prod_{k=1}^{\infty}\left(1+\frac{f_{j}^{2}}{\pi^{2}(2 k)^{2}}\right) \\
\cosh \frac{f_{j}}{2} & =\prod_{k=0}^{\infty}\left(1+\frac{f_{j}^{2}}{\pi^{2}(2 k+1)^{2}}\right),
\end{aligned}
$$

the points $a$ entering into the right-hand side of formula (5.5) have to satisfy a system (7.1) with $c_{j}=\pi k_{j}$, where $k=\left(k_{1}, \ldots, k_{n}\right) \in \mathbb{Z}^{n}$. Denote by $N_{f-\pi k \imath}$ the set of roots of the system $f(z)=\pi k \imath$. Corollary 6.1 yields

$$
I(s)=\frac{\pi^{n}}{\sin \pi\left(1-s_{1}\right) \cdot \ldots \cdot \sin \pi\left(1-s_{n}\right)} \sum_{k \in \mathbb{Z}^{n}} \sum_{a \in N_{f-\pi k \imath}}(-1)^{o(k)}(-a)^{-s},
$$

where $o(k)$ is the number of odd components of the multi-index $k$.

Acknowledgements This article was written during the stay of first two authors at the Institute of Mathematics, University of Potsdam. They gratefully acknowledge 
the financial support of the Deutsche Forschungsgemeinschaft and the RFFI grant 02-01-00167. 


\section{REFERENCES}

[AYu83] L. A. Aizenberg and A. P. Yuzhakov, Integral Representations and Residues in Multidimensional Complex Analysis, Transl. Math. Monographs, AMS, Providence, RI, 1983.

[BKL98] V. Bykov, A. Kytmanov, and M. Lazman, Elimination Methods in Polynomial Computer Algebra, Kluwer Academic Publishers, Dordrecht, 1998.

[Dik55] L. A. DikiI, Zeta-function of an ordinary differential equation on a finite interval, Izv. Akad. Nauk SSSR, Ser. Mat. 19 (1955), 187-200.

[Dik58] L. A. DikII, Trace formulas for Sturm-Liouville differential operators, Uspekhi Mat. Nauk 13 (1958), no. 3, 111-143.

[GL53] I. M. GELFAND and B. M. LeVitan, On a simple identity for the characteristic values of a differential operator of the second order, Doklady Akad. Nauk SSSR 88 (1953), 593-596.

[Lev64] B. M. LEvitAn, Calculation of the regularized trace for the Sturm-Liouville operator, Uspekhi Mat. Nauk 19 (1964), no. 1 (115), 161-165.

[LS67] V. M. Lidskit and V. A. SAdovnichi, Regularized sums of roots of a class of entire functions, Funkts. Analiz 1 (1967), no. 2, 52-59.

[LS68a] V. M. Lidskir and V. A. SAdovnichir, Asymptotic formulas for the roots of a class of entire functions, Mat. Sb. 75 (117) (1968), 558-566.

[LS68b] V. M. Lidskil and V. A. SAdovnichiI, Trace formulas in the case of the OrrSommerfeld equation, Izv. Akad. Nauk SSSR, Ser. Mat. 32 (1968), 633-648.

[See69] R. T. SEeley, Analytic extension of the trace associated with elliptic boundary problems, Amer. J. Math. 91 (1969), 963-983.

[Shu87] M. A. Shubin, Pseudodifferential Operators and Spectral Theory, Springer-Verlag, Berlin et al., 1987.

[Tsi92] A. K. TsikH, Multidimensional Residues and Their Applications, Transl. Math. Monographs, AMS, Providence, RI, 1992.

(Alexander Kytmanov) Krasnoyarsk State University, Pr. Svobodnyi 79, 660041 KrasNOYARSK, RUSSIA

E-mail address: kytmanov@lan.krasu.ru

(Simona Myslivets) Krasnoyarsk State University, Pr. Svobodnyi 79, 660041 KrasnoYARSK, RUSSIA

E-mail address: simona@lan.krasu.ru

(Nikolai Tarkhanov) Universität Potsdam, Institut für Mathematik, Postfach 601553 , 14415 Potsdam, Germany

E-mail address: tarkhanov@math.uni-potsdam.de 